\title{
Light-Emitting Transistor Based on Real-Space Transfer: Electrical and Optical Properties
}

\author{
Marco Mastrapasqua, Member, IEEE, Serge Luryi, Fellow, IEEE, Federico Capasso, Fellow, IEEE, \\ Albert L. Hutchinson, Deborah L. Sivco, and Alfred Y. Cho, Fellow, IEEE
}

\begin{abstract}
The charge injection transistor is implemented in InGaAs/InAIAs/InGaAs heterostructure material, grown by molecular beam epitaxy. A complementary collector of p-type conductivity is used for the first time. The real-space transfer of hot electrons leads to a luminescence signal proportional to the injection current. The radiative efficiency is significantly enhanced by a double-heterostructure design of the collector active region, which confines the injected minority carriers. The internal quantum efficiency of the light-emitting transistor is comparable to that of light-emitting diodes. Due to a peculiar symmetry of real-space transfer, the optical output signal follows an exclusive-OR function of input voltages. Functional logic operation of the device is demonstrated at room temperature.
\end{abstract}

\section{INTRODUCTION}

$\mathrm{T}$ HE CONCEPT of real-space transfer (RST) [1], [2] describes the process in which electrons in a narrow $(\leq 0.1 \mu \mathrm{m})$ semiconductor layer are heated by a parallel electric field and spill over an energy barrier into the adjacent layer. This principle underlies the operation of a three-terminal hot-electron device, called the charge-injection transistor or CHINT [3], [4]. The RST in CHINT occurs between two independently contacted conducting layers. One of these layers, "emitter," has source and drain contacts and plays the role of a hot-electron cathode. The other conducting layer ("collector") is separated by a heterostructure barrier. Transistor action consists in the control of the injection current $I_{C}$ by the voltage $V_{D S}$, applied between the drain and the source.

Charge injection transistors and related devices have been widely studied both theoretically and experimentally. 'All previously studied samples had the same type of conductivity in the emitter channel and the collector. However, the RST of carriers into a complementary collector layer allows the implementation of light-emitting devices endowed with a logic functionality with respect to electrical input. A complementary CHINT with a properly designed optically active layer structure in the collector has been predicted [5] to produce an optical output

Manuscript received June 4, 1992. M. Mastrapasqua was supported by the Associazione Elettrotecnica ed Elettronica Italiana under E. De Castro fellowship. The review of this paper was arranged by Associate Editor M. Shur

The authors are with AT\&T Bell Laboratories, Murray Hill, NJ 07974 IEEE Log Number 9205577.

'General references on real-space transfer devices can be found in the review [4]
$L_{\text {OUT }}$ that is proportional to the RST current and obeys an exclusive-OR function of the input signals $L_{\mathrm{OUT}}=$ xor $\left(V_{S}, V_{D}\right)$.

In this work we report the first realization of a CHINT with an n-type emitter channel and a p-type collector (a brief preliminary account has been reported earlier [6]). Both the electrical and optical properties are discussed in detail. The light-emitting device operates at room temperature and exhibits a high radiative efficiency of RST electrons as well as a high light-power on/off ratio. The expected logic functionality is demonstrated both in the electrical and the optical outputs. Interestingly, the optical logic performance turns out to be superior compared to the electrical performance, especially at higher temperatures. This we explain by analyzing the leakage current and showing that, in contrast to the RST current, it is mainly nonradiative. Our study suggests important considerations for the design of RST optoelectronic devices.

\section{Structure}

The complementary CHINT has been implemented in a lattice-matched InGaAs / InAlAs heterostructure, grown by molecular beam epitaxy on a semi-insulating InP substrate. A schematic cross section of the device is illustrated in Fig. 1. The design is based on the epitaxial contact scheme, first used in the fabrication of CHINT by Mensz et al. [7]. In this scheme, the channel length is defined by a trench etched in the $\mathrm{n}^{+}$cap layer $(a)$, while the remaining portions of the cap layer make ohmic contacts to the source and drain metal. The $25-\AA$ InAlAs layer (b) is used as an etch stop in the selective etching of the cap layer [8]. The collector contact, $\mathrm{AuBe} / \mathrm{Au}$, has been alloyed at $450^{\circ} \mathrm{C}$ for $10 \mathrm{~s}$ to ensure a sufficient penetration into the InAlAs collector confinement layer.

All patterns, including the trench between source and drain, were defined by standard optical contact lithography. The emitter channel length, defined by the trench, is $L_{\mathrm{ch}}=3 \mu \mathrm{m}$ and the width $W=50 \mu \mathrm{m}$. The design, aimed at ease of processing, made no attempt to minimize parasitic capacitances and improve the device speed. For this reason, only dc characterization has been carried out.

Fig. 2 shows a schematic energy-band diagram of the device in a cross section under the trench. In equilibrium, Fig. 2(a), the emitter channel is entirely depleted by the surface potential and the $\mathrm{p}-\mathrm{n}$ junction, so that the channel 


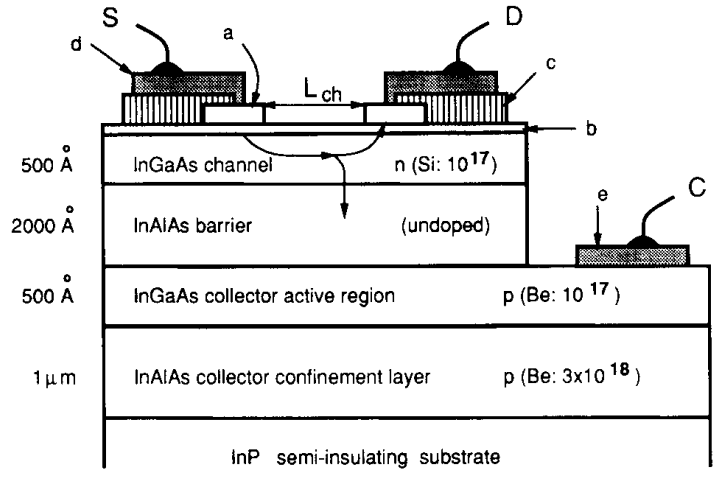

$$
\begin{array}{ll}
\text { a: } 200 \AA \operatorname{lngaAs}, n\left(\mathrm{Sn}: 10^{20}\right) & \text { d: } 300 \AA \text { Ti } / 1800 \AA \mathrm{Au} \\
\text { b: } 25 \AA \ln A l A s, n\left(\mathrm{Sn}: 10^{19}\right) & \text { e: } 800 \AA \text { AuBe } / 2000 \AA \mathrm{Au} \\
\text { c: } 2500 \AA \mathrm{Si}_{3} \mathrm{~N}_{4} &
\end{array}
$$

Fig. 1. Cross section of the sample structure. The real-space transfer current is indicated by the downward arrow.

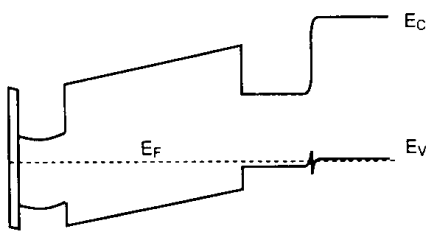

(a)

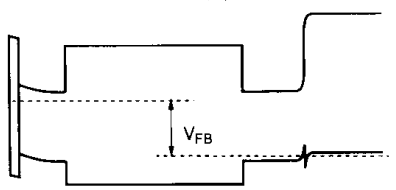

(b)

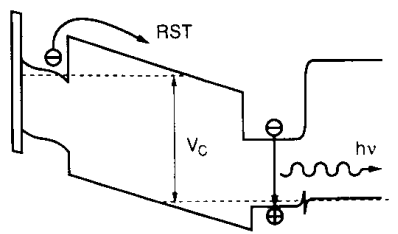

(c)

Fig. 2. Schematic energy-band diagram in a device cross section under the trench. (a) Equilibrium. The collector and the emitter-channel layers form a $p-n$ junction, separated by a wide-gap barrier. The channel is depleted by the surface potential. (b) Flatband condition. The flatband voltage $V_{C}=$ $V_{F B}$ is undetermined because of the uncertainty in the surface potential. The calculated flatband voltage in a cross section under the contacts is $V_{F B}=$ $0.63 \mathrm{~V}$. (c) Operating regime of a complementary CHINT. The channel charge is induced by the collector field. When the heating electric field is applied along the channel, the latter becomes a hot-electron emitter. Realspace transferred electrons radiatively recombine with holes in the active region of the collector.

conduction requires a positive collector voltage to induce a two-dimensional electron gas at the interface with the barrier. In the "flatband" condition, illustrated in Fig. 2(b), the collector bias $V_{C}=V_{F B} \approx 0.6 \mathrm{eV}$ corresponds to the work-function difference between $n$-InGaAs in the channel and $\mathrm{p}$-InGaAs in the collector. In the operating regime, Fig. 2(c), the p-n junction is forward-biased and the main obstacle to current is due to the band discontinuities at the two interfaces with the InAlAs barrier. Inasmuch as the valence-band discontinuity in the InGaAs / InAlAs heterosystem is smaller than the conduction band discontinuity [9] $\left(\Delta E_{V}=0.20 \mathrm{eV}<\Delta E_{C}=\right.$ $0.50 \mathrm{eV}$ ) we can expect that, in the absence of RST. the collector current will be dominated by holes.

Electron heating is generated by a drain-to-source bias $V_{D S}$. The real-space transfer manifests itself in the increasing collector current $I_{C}$. As usual, it is accompanied by a negative differential resistance in the drain current $I_{D}$. In our complementary structure, the RST is also accompanied by a recombination radiation from the $\mathrm{p}$-type collector.

The purpose of the wide-gap $\mathrm{p}^{+}$InAlAs layer in the collector is to spatially confine electrons injected over the barrier and, at the same time, to provide a low-resistance path for the collector current. Both requirements are very important. Confinement is necessary for the radiative efficiency (otherwise, most of the injected electrons would reach the collector contact prior to recombination) and low collector resistance is necessary to minimize a spurious backgating effect, which would otherwise severely limit the available RST current.

\section{Electrical Characterization}

Electrical properties of a complementary CHINT are quite different from those of unipolar devices studied previously. Most of the differences are rooted in the fact that the collector and the emitter form a $p-n$ junction with a wide-gap barrier in between. The device operating regime corresponds to a forward bias of the $p-n$ junction, hence we must rely exclusively on the bandgap discontinuities to block the unwelcome leakage current. In what follows we shall refer to as the leakage that part of the collector current which flows independently of the electron temperature $T_{c}$ controlled by the lateral field in the emitter channel. Identification of the leakage mechanism is an important part of the device characterization.

\section{A. Leakage Current}

In a unipolar CHINT device, the leakage can be strongly suppressed by choosing a heterostructure with a large conduction band discontinuity. Excellent results have been obtained [8] in the InGaAs/InAlAs system which has $\Delta E_{C}=0.50 \mathrm{eV}$. In a complementary CHINT, we have to worry about the flux of holes from the collector into the emitter channel.

Fig. 3 shows the collector leakage current, with both source and drain grounded, as a function of the collector bias $V_{C}$ for different temperatures. At high temperatures, $200 \leqslant T \leqslant 300 \mathrm{~K}$, and relatively low bias the current obeys the thermionic model

$$
I_{C}=I_{C}^{(0)} e^{q V_{C} / n k T}
$$

where $n$ is the ideality factor and $I_{C}^{(0)}$ the diode saturation current. From the slopes of the $I_{C}\left(V_{C}\right)$ curves at low bias, 


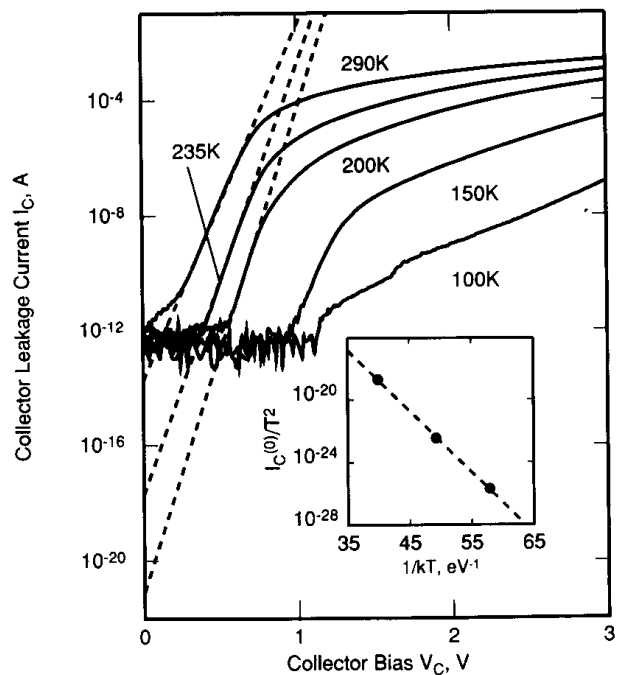

Fig. 3. Collector current-voltage characteristics (source and drain grounded) at different temperatures. Dashed lines indicate the linear extrapolation of $I_{C}$ to zero forward bias. The intercepts of the dashed lines with the ordinate axis correspond to $I_{C}^{(0)}$. Inset shows an Arrhenius plot of $I_{C}^{(0)} / T^{2}$.

indicated by the dashed lines in Fig. 3, we determine $n \approx 1.4$. Extrapolating to $V_{C}=0$, we determine $I_{C}^{(0)}$ and find that it can be fitted to the usual form

$$
I_{C}^{(0)}=S A^{*} T^{2} e^{-\Phi / k T}
$$

where $S=1.2 \times 10^{-5} \mathrm{~cm}^{2}$ is the total emitter area including the source and drain contacts, $\Phi$ is the barrier height at zero bias (separation between the hole Fermi level in the collector and the valence band edge in the barrier at the channel interface), and $A^{*}$ the effective Richardson constant. It is worth noting that the actual trench area $\left(1.5 \times 10^{-6} \mathrm{~cm}^{2}\right)$ is smaller than $S$; moreover, at low collector bias the areas under the trench and the $\mathrm{Si}_{3} \mathrm{~N}_{4}$ are depleted and contribute little current. Most of the current flows therefore in regions under the source and drain contacts, which are not depleted even in equilibrium.

The inset to Fig. 3 shows an Arrhenius plot of $I_{C}^{(0)} / T^{2}$ versus $T^{-1}$ in the high-temperature range. The slope of this plot gives $\Phi=0.90 \mathrm{eV}$ in good agreement with a calculated value $\Phi^{h}$ of the barrier height for holes relative to their Fermi level in the collector, $\Phi^{h}=0.89 \mathrm{eV}$, based on the energy gap $E_{G}=0.75 \mathrm{eV}$ of InGaAs, the valenceband discontinuity $\Delta E_{V}=0.20 \mathrm{eV}$, and given doping levels. This is to be contrasted with the estimated zero-bias barrier height for emitter electrons, $\Phi^{e}=1.10 \mathrm{eV}$. Moreover, extrapolating $\log \left[I_{C}^{(0)} / T^{2}\right]$ to $T^{-1} \rightarrow 0$, we find $A^{*}$ $\approx 63 \mathrm{~A} \cdot \mathrm{cm}^{-2} \cdot \mathrm{K}^{-2}$ in agreement with the theoretical value for heavy holes. This indicates that our data in Fig. 3 can be used for a rather good estimate of the valenceband discontinuity in the $\operatorname{In}_{0.53} \mathrm{Ga}_{0.47} \mathrm{As} / \mathrm{In}_{0.52} \mathrm{Al}_{0.48} \mathrm{As}$ system, giving $\Delta E_{V} \approx 0.21 \mathrm{eV}$.

At higher collector biases, exceeding the flatband condition $V_{C}>V_{F B}$, the top of the barrier for holes is at the collector interface. This is the operating regime of a complementary CHINT. Further increase of the collector current with $V_{C}$ occurs primarily because of the accumulation of holes (increasing the Fermi level) and also, because of the increasing role of hole tunneling which lowers the effective barrier. Hence the leakage curve departs from (1) when $V_{C}>V_{F B}$. As is evident from Fig. 3, in the operating regime the slope of $\log I_{C}\left(V_{C}\right)$ is relatively gentle. At low temperatures, $T \leqslant 150 \mathrm{~K}$, thermally assisted tunneling of holes is the dominant leakage mechanism.

\section{B. Transistor Characteristics}

The usual way of presenting current-voltage characteristics of a charge injection transistor is to plot the family of $I_{C}\left(V_{D}\right)$ and $I_{D}\left(V_{D}\right)$ curves at constant values of $V_{C}$. Fig. 4(a)-(c) shows such characteristics of the complementary CHINT at $T=290 \mathrm{~K}, T=235 \mathrm{~K}$, and $T=100 \mathrm{~K}$. The low $V_{D}$ part of the characteristics is similar to that in a "normally-off" field-effect transistor (FET), with the collector playing the role of a gate. At a fixed $V_{C}$ and increasing the heating voltage $V_{D}$, the channel current first $\left(V_{D} \leqslant 0.5 \mathrm{~V}\right)$ increases linearly, then sublinearly with a tendency for saturation. The saturation value of the current increases with $V_{C}$, as it would in a FET. When the heating voltage is high enough to establish a significant RST, the drain current shows a negative differential resistance (NDR). The NDR effect is enhanced at lower temperatures, where it manifests itself by an abrupt drop in $I_{D}$. Simultaneously, the collector current rapidly increases from its leakage value.

Note that the increase in $I_{C}$ does not fully compensate for diminishing $I_{D}$, which means, by Kirchhoff 's law, that the source current $I_{S}$ also decreases. This behavior can be explained by two mechanisms, both due to the backgating effect of the collector field. The first mechanism, which might be termed intrinsic, is due to the charge dynamically stored in the barrier layer in transit toward the collector [10]. The negative dynamic charge partially screens the collector field and reduces the electron concentration in the emitter channel. The second mechanism is due to a negative feedback of the collector current in the presence of a parasitic series resistance in the collector path. When the RST current begins to flow, the voltage drop on that series resistance reduces the collector potential, effectively lowering the $V_{C}$. Both effects result in decreasing $I_{S}$. When the collector contact is not properly alloyed, the series resistance may dominate. ${ }^{2}$ In our device the dynamic space-charge effect may account for most of the decrease in the source current, as can be ascertained by simple estimates similar to those in [10]. Nevertheless, a residual collector series resistance $\left(R_{C} \leqslant 0.5 \mathrm{k} \Omega\right.$ at 290 $\mathrm{K}$ but higher at lower temperatures, see below) is likely to be present in our structure; without $R_{C}$ we find it hard to understand certain features in the characteristics at high $V_{D}$ and still higher $V_{C}$.

Such structures usually show a giant NDR accompanying a relatively little RST current. This anomaly goes away upon annealing the alloyed contact 


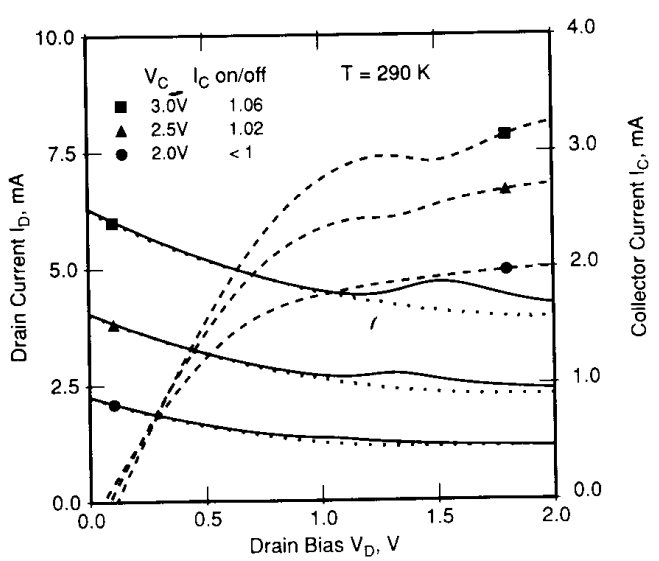

(a)

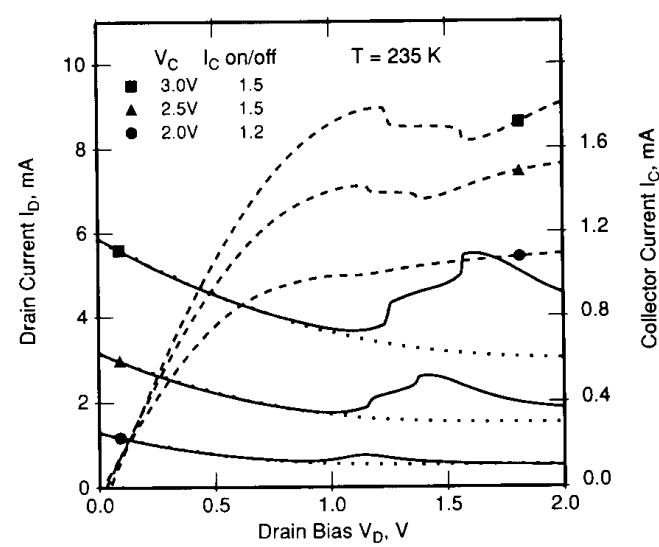

(b)

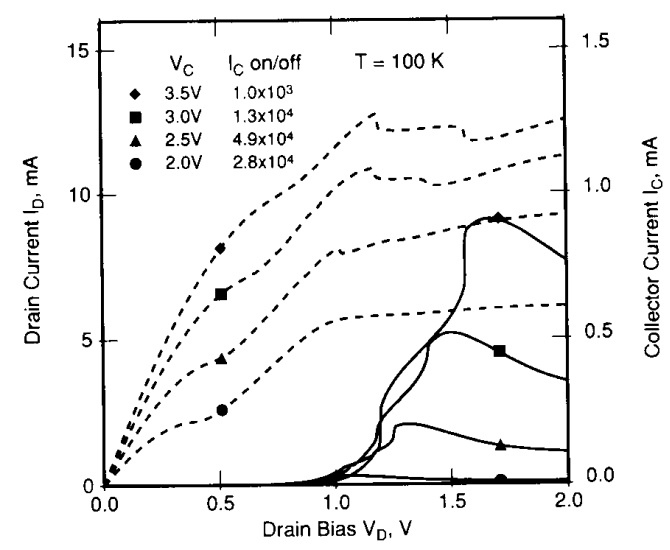

(c)

Fig. 4. Current-voltage characteristics at different temperatures and collector biases $V_{C}$. Drain current $I_{D}$ is shown by the dashed lines and collector lector biases $V_{C}$. Drain current $I_{D}$ is shown by the dashed lines and collector
current $I_{C}$, by the solid lines. The dotted lines show the expected leakage behavior of $I_{C}$ in the absence of RST, calculated from Fig. 3 . The collector current on/off ratio is calculated by dividing the (relative) maximum value of $I_{c}$ by its minimum value before the onset of real-space transfer. (a) $T=$ $290 \mathrm{~K}$. (b) $T=235 \mathrm{~K}$. (c) $T=100 \mathrm{~K}$

Indeed, as seen from Fig. 4, the collector current shows a well-defined maximum as a function of $V_{D}$. This behavior is faithfully tracked by the electroluminescence signal, which, as discussed in Section IV, indicates that the maximum is associated with a decrease of the electron RST current at high $V_{D}$. We believe, that the existence of a peak in the collector current and the decrease of $I_{C}$ at higher $V_{D}$ is related to the increase in the potential of the "hot spot" (i.e., the high-field region in the channel where most of the RST occurs) relative to the collector. Reversal of the field in the barrier at the hot spot exponentially suppresses the RST. No peak in $I_{C}$ is observed when $V_{D}$ is increased simultaneously with increasing the collector voltage, which corresponds to varying the source voltage at fixed $V_{D}$ and $V_{C}$. Field reversal at the hot spot naturally explains our data in Fig. 4 at lower $V_{C} \leq 2.5$ V. However, a close examination of Fig. 4(c) shows that it does not work directly for higher values of $V_{C}$. Thus for $V_{C}=3.5 \mathrm{~V}$, the maximum occurs near $V_{D}=1.7 \mathrm{~V}$. In the absence of a collector resistance, this would suggest that the entire emitter channel is in the operating regime and no field reversal takes place at the hot spot. To make the ends meet, it is sufficient to assume a series resistance $R_{C} \approx 1 \mathrm{k} \Omega$ in the collector circuit at low temperatures.

Prior to the onset of the RST, the collector current is seen to decrease, cf., the higher temperature plots, Fig. $4(a)$ and (b). This is due to the diminishing leakage current (roughly by a factor of 2) with decreasing collectorto-drain bias. The same behavior occurs at low temperatures, but on the scale of Fig. 4(c) the leakage is not resolved.

We would like to distinguish between two contributions to the measured collector current

$$
I_{C}=I_{C}^{\mathrm{LKG}}+I_{C}^{\mathrm{RST}}
$$

where $I_{C}^{\mathrm{RST}}$ is the real-space transfer current of hot electrons and $I_{C}^{\mathrm{LKG}}$ is the collector leakage, defined as the current which would flow at a given voltage configuration $\left(V_{C}, V_{D}\right)$ if the electron heating phenomenon were absent. at low temperatures, $I_{C}^{\mathrm{LKG}}$ is negligible and $I_{C}^{\mathrm{RST}}$ is very close to the measured $I_{C}$. On the other hand, in order to estimate the $I_{C}^{\mathrm{RST}}$ at high temperatures it is necessary to subtract the leakage contribution. The leakage curves, $I_{C}^{\mathrm{LKG}}\left(\boldsymbol{V}_{D}\right)$, estimated from the diode characteristics of Fig. 3 (assuming a perfect symmetry between the source and the drain), are plotted by the dotted lines in Fig. 4(a) and (b). Subtracting the leakage curve, we obtain a reasonable approximation to the RST current $I_{C}^{\text {RST }}$ at a given bias. Thus calculated curves $I_{C}^{\mathrm{RST}}\left(V_{D}\right)$ are plotted in Fig. 5 below.

\section{Optical Characterization}

Electroluminescence of the complementary CHINT was detected from the back of the polished substrate using a liquid-nitrogen-cooled $\mathrm{Ge}$ detector and a $0.75-\mathrm{m}$ spectrometer. We observed a spectrum peaked at the photon energy corresponding to the band-to-band recombination in InGaAs. As the bandgap shrinks with increasing temperature, the peak wavelength increases from $1.56 \mu \mathrm{m}$ at $T=100 \mathrm{~K}$ to 1.59 and $1.60 \mu \mathrm{m}$ at 235 and $290 \mathrm{~K}$, re- 


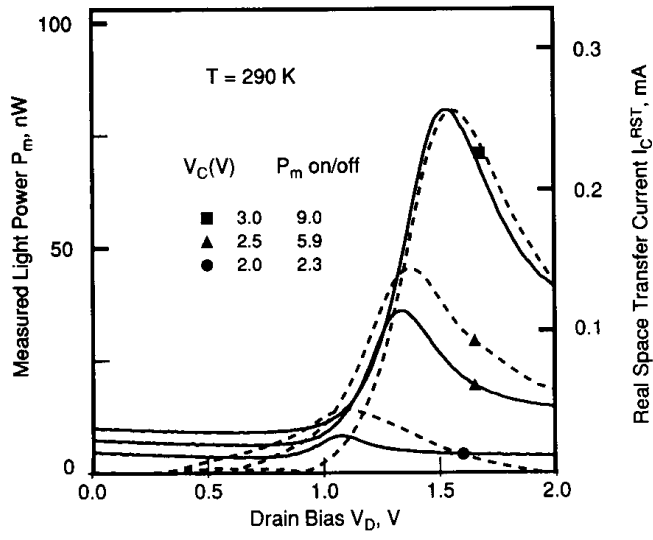

(a)

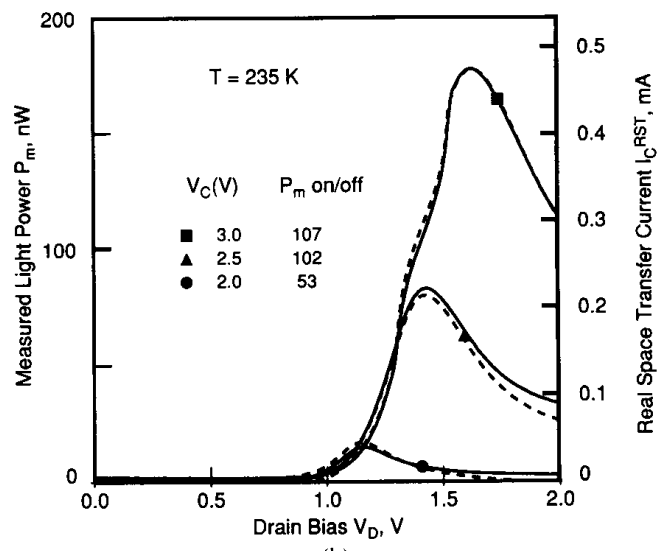

(b)

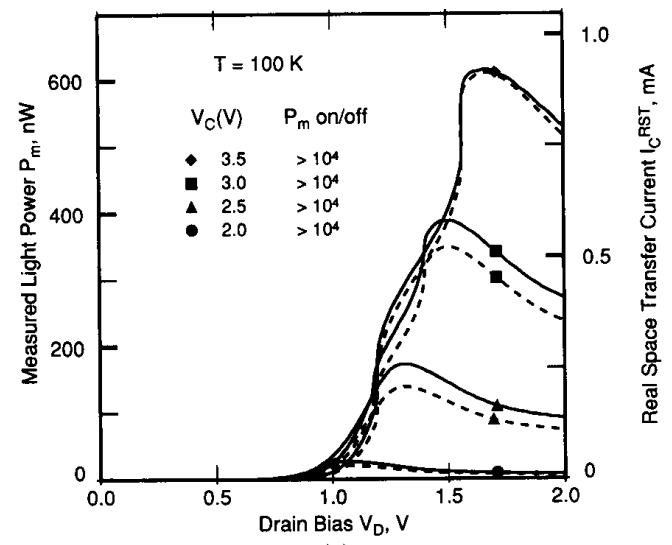

(c)

Fig. 5. Optical output characteristics versus the heating voltage $V_{D}$ at different temperatures and collector biases $V_{C}$. The total measured light power $P_{m}$ is plotted by solid lines. Dashed lines indicate the real-space transfer current $I_{C}^{\mathrm{RST}}$ calculated from the measured $I_{C}$ by subtracting the leakage current. The current measurements were taken simultaneously with the optical data and differ slightly from those presented in Fig. 4. The light power on/off ratio is calculated by dividing the maximum value of $P_{m}$ by its minimum value before the onset of real-space transfer. (a) $T=290 \mathrm{~K}$. (b) $T$ $=235 \mathrm{~K}$. (c) $T=100 \mathrm{~K}$.

spectively. Moreover, at low temperatures $(\$ 100 \mathrm{~K})$ the position of the peak slightly shifts towards higher energy as the RST current increases. We attribute this shift to a band-filling effect due to the increasing electron injection. To measure the total emitted power, we used a broad-area Ge photodiode and suitable focusing optics.

Fig. 5 shows the dependence of the measured light power $P_{m}$ on the electron heating voltage $V_{D}$ at selected temperatures and different values of $V_{C}$. On the same graphs we show the "net" RST curves $I_{C}^{\text {RST }}\left(V_{D}\right)$, calculated as described in Section III-B. The first notable observation is that the $P_{m}\left(V_{D}\right)$ curves are to a good degree proportional to $I_{C}^{\mathrm{RST}}\left(V_{D}\right)$, rather than to the total $I_{C}\left(V_{D}\right)$ of Fig. 4, as one could naively expect. Accordingly, the measured optical on/off ratio (Fig. 5) far exceeds the on/ off ratio in the collector current, the difference being particularly striking at low temperatures. Even at $T=290$ $\mathrm{K}$, where the RST structure in $I_{C}\left(V_{D}\right)$ is barely visible against the leakage background, the corresponding modulation of the optical signal is by an order of magnitude: for $V_{C}=3.0 \mathrm{~V}$ the on/off ratio is 9 in $P_{m}$ and 1.1 in $I_{C}$. The origin of this remarkable performance must be clearly understood, since the depth of modulation is of paramount importance for room-temperature logic applications of the light-emitting CHINT.

By studying the temperature dependence of $I_{C}\left(V_{C}\right)$ at $V_{D}=0$, we had concluded in Section III-A that the collector leakage is mostly due to the injection of holes into the channel. Now, the data of Fig. 5 clearly indicate that this injection does not contribute to the optical signal. It is significant that the leakage and the RST are caused by different types of carriers. The injected holes have a vastly lower radiative efficiency because they are likely to reach the source or drain contact before they recombine radiatively with electrons. The $\operatorname{In}_{0.52} \mathrm{~A}_{10.48} \mathrm{As}$ etch-stop layer $b$ (Fig. 1) is insufficiently thick to confine minority holes in the emitter channel (the estimated hole escape time by tunneling across this layer is much shorter than the radiative recombination time). Most of the nonradiative recombination is likely to occur in the layers heavily doped with tin. In contrast, electrons injected into the collector are confined in the active region. Virtually all of the optical output can be attributed to the recombination of injected electrons in the collector active layer. This implies an important design consideration: to maximize the optical on/off ratio it is essential to suppress the leakage of electrons into the active region; the oppositely directed flux of holes can be tolerated.

At low temperatures, Fig. 5(c), the light signal related to the leakage is below the noise level and the on/off ratio in $P_{m}$ is above $10^{4}$. At higher temperatures, the small electron component of the leakage current increases and consequently the on/off ratio disparity between the lightpower output and the collector current is less pronounced. From the measured light power $P_{m}\left(V_{D}, V_{C}\right)$ we can estimate the internal radiative efficiency of the RST electrons as follows:

$$
\eta_{q}=\frac{1}{h \nu \eta_{c} t_{o}} \frac{q \Delta P_{m}}{I_{C}^{\mathrm{RST}}}
$$

where $\eta_{c} \approx 0.48 \%$ is the collection efficiency (estimated 
assuming an isotropic emission over the $2 \pi$ solid angle. due to reflection from the surface metallization), $t_{t} \approx 88 \%$ is the combined transmission of the optical components (lens and windows) between the detector and the device, and $h \nu$ is the photon energy at the peak of the luminescence spectrum. To account for the light power due to the leakage current, the quantity $\Delta P_{m}$ is taken equal to $P_{m}\left(V_{D}\right.$, $V_{C}$ ) minus the optical power measured at the same $V_{C}$ but lower $V_{D}$, prior to the onset of the RST. At lower temperatures, one has $\Delta P_{m}=P_{m}$.

The fact that our collection efficiency $\eta_{\text {c }}$ is low, is accounted for by the total internal reflection, Fresnel loss, and the collection solid angle. We have not used an antireflection coating. The relatively low numerical aperture $(N A \approx 0.34)$ has been forced by the use of a dewar for low-temperature measurements. Moreover, an error of $\pm 5 \%$ in $N A$ from the collection lens being slightly out of focus, produces an error of $+10 \%$ in $\eta_{c}$. Nevertheless, the systematic error in the determination of the efficiency was the same for all measurements, since the lens system was not adjusted during the whole set of measurements. The relative efficiency behavior at different temperatures and biases can be considered quite reliable.

Fig. 6 shows the basic trends in the behavior of the radiative efficiency $\eta_{\varphi}$ under varying temperature and collector bias. We found that near and above the peak of the RST current the efficiency is not modulated by varying $V_{D}$. This means that $\eta_{q}$ is practically independent of the injection current at a given $T$ and $V_{C}$. The data in Fig. 6 are taken near the RST peak; the dependence on $V_{D}$ in a wider range is discussed below. As is evident from the figure, the radiative efficiency decreases with increasing temperature. This dependence is likely to be explained, as in conventional light-emitting devices, by the increasing role at higher $T$ of nonradiative processes, such as Auger recombination [11].

The radiative efficiency is also seen to decrease with increasing collector bias. This effect is prominent only at low $T \leqslant 150 \mathrm{~K}$ (no significant variation $\eta_{q}$ with $V_{C}$ is observed at higher temperatures; the apparent dependence at $T \geq 200 \mathrm{~K}$ in Fig. 6 is not meaningful since the low$V_{C}$ points are within an experimental error introduced by the uncertainty in the subtraction of the leakage component). One possible explanation for the $V_{C}$ dependence may be related to the injection of hot electrons into the wide-gap collector confinement layer, where they recombine nonradiatively. ${ }^{3}$ Within this model, weakening of the $V_{C}$ dependence of $\eta_{4}$ at higher temperatures can be explained by a shorter hot-electron mean-free path due to higher rate of optical-phonon scattering. The fact that a sizable fraction of injected electrons can retain enough energy (especially at lower temperatures) to clear the sec-

\footnotetext{
Even if there were a radiative component from the confinement layer. it would not be detected experimentally. since all of the InAlAs light output would be re-absorbed in the InP substate. Moreover, as a matter of principle. one would not expect a significant radiation from the wide-gap layer. since electrons there are no longer contined and their radiative lifetime is longer than the travel time to the contact.
}

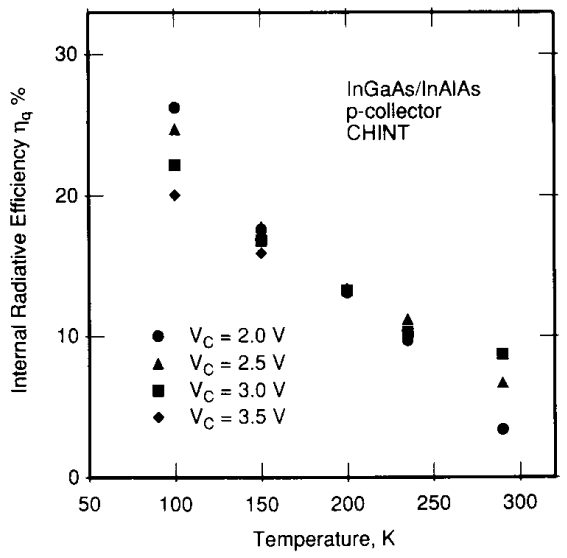

Fig. 6. Dependence of the internal radiative efficiency $\eta_{q}$ on the lattice temperature for different collector biases.

ond barrier, is well known from studies of ballistic transistors (see, for example, [12] and the literature cited therein). This interpretation does not contradict the fact that our measured spectra do not show hot-electron tails, because the electron cooling rates in InGaAs are known to be faster than recombination [13]. In our preliminary report [6], the "ballistic-transistor" model was mentioned as a possible explanation of the $V_{C}$ dependence.

Nevertheless, we have difficulties with this interpretation. First, it is inconsistent with our understanding of the peak in the $I_{C}\left(V_{C}\right)$ dependence, which we had attributed in Section III-B to a field reversal in the "hot-spot" region of the channel. Clearly, one would expect a suppression of the ballistic-transistor effect in this case. This contradicts our data in Fig. 7, which shows the dependence of the calculated $\eta_{q}$ on the heating voltage $V_{D}$ for several values of $V_{C}$ at $T=100 \mathrm{~K}$. No enhancement of the $\eta_{G}$ occurs at the heating voltages $V_{D}$ above the $I_{C}$ peak.

Next, consider the most striking feature of Fig. 7; namely, the dramatic downward steps in the efficiency, accompanying steps in the collector current. The low-bias values of the efficiency are relatively high and largely independent of the collector voltage. ${ }^{4}$ As the heating bias reaches the location of the first current step, the efficiency suddenly drops to a lower plateau value. Moreover, curves corresponding to lower collector biases $\left(V_{C}=2.0\right.$ and 2.5 $\mathrm{V})$, at which there are no steps in the current-voltage characteristics, show no significant decrease in efficiency either. For $V_{C}>2.5 \mathrm{~V}$, something evidently happens near $V_{D} \approx 1.2 \mathrm{~V}$ which forces the step-like switching of the current and is accompanied with the efficiency drop. Steps in the current-voltage characteristics of the CHINT are usually explained [7], [14], [15] by an instability associated with the formation of hot-electron domains in the

${ }^{1}$ The apparent decrease in $\eta_{4}$ at lowest heating biases (near the sensitivity limit of our light detector) is probably related to non radiative recombina tion with defects. The rate of such processes scales linearly with the minority concentration and at low carrier injection it exceeds the radiative recombination rate 


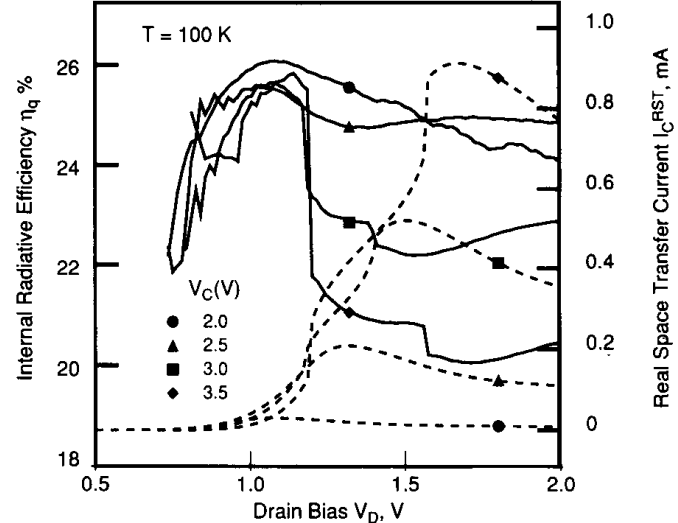

Fig. 7. Dependence of the internal radiative efficiency $\eta_{4}$ on the heating voltage $V_{D}$ at $T=100 \mathrm{~K}$ and different collector biases. Dashed lines indicate the RST collector current (from Fig. 5).

channel. Both the field and the electron temperature $T_{e}$ rise dramatically in the domains and that, of course, may lead to a higher injection into the confinement layer, because of the higher average energy of injected electrons. However, if the injection energy is the deciding factor, then we should expect the radiative efficiency go down strongly with increasing $V_{C}$ at all values of $V_{D}$. The trouble with the above ballistic-transistor interpretation of the $\eta_{q}\left(V_{C}\right)$ dependence is not that we cannot stretch it to explain the efficiency drop (that we can do!) but that it is clearly inconsistent with the fact that low- $V_{D}$ values of $\eta_{q}$ are practically independent of $V_{C}$. We are, therefore, led to consider alternative interpretations of the efficiency data.

In our opinion, a possible explanation involves a nonlocal heating of the lattice by hot electrons. In this picture, the lattice temperature in the active layer may substantially deviate from the ambient temperature, due to the power $W_{e}$ dissipated by hot electrons in the channel via optical-phonon emission. This power can be estimated as

$$
W_{e}=\mathfrak{Q} \frac{n k T_{e}}{\tau_{E}}
$$

where $n$ is the sheet carrier concentration in the emitter channel at the hot spot, $Q$ the hot-spot area, and $\tau_{E}\left(T_{e}\right)$ the energy relaxation time of hot electrons at temperature $T_{e}$. At low $V_{D}$ the heating field in the channel is low and relatively uniform, hence $T_{e}$ is low and so is $W_{e}$. The formation of a hot-electron domain shrinks $Q$ but at the same time strongly increases $T_{e}$ and therefore $W_{e}$ goes up. Higher phonon emission in the channel leads to a higher lattice temperature in the collector active layer and hence a lower radiative efficiency. From the data of Fig. 6, the required temperature increase at $T=100 \mathrm{~K}$ is by less than $50 \mathrm{~K}$. The dependence on $V_{C}$ results from the field-effect gating action of the collector, expressed in (5) by the factor $n\left(V_{C}\right)$. After the domain is formed, the dependence on $V_{D}$ is weak.
We would like to stress that the local heating by hot carriers is an essential part of this picture. A simple Joule heating of the whole device is ruled out by the following observation. Consider the total input power $W_{\text {tot }}$ into the transistor, which in the common-source configuration is given by

$$
W_{\mathrm{tot}}=I_{C} V_{C}+I_{D} V_{D}
$$

To our surprise, we found that $W_{\text {tot }}$ is continuous at the steps in the current-voltage characteristics. ${ }^{5}$ To avoid misunderstanding, let us remark that $W_{e}$ is only a part of $W_{\text {tot }}$. Most of $W_{\text {tot }}$, however, is dissipated by acoustic phonons and distributed over a large volume. In contrast, the power $W_{e}$, dissipated by electrons with $T_{e}>\sim 1000 \mathrm{~K}$, goes into the generation of relatively immobile optical phonons at the hot spot.

\section{Real-Space Transfer Logic}

A fundamental property [15]-[17] of the CHINT consists in a symmetry equivalence between the internal states $\delta\left[V_{D}, V_{C}\right]$ of the device at the external biases

$$
\mathcal{S}\left[V_{D}, V_{C}\right] \stackrel{S}{ }\left[-V_{D},\left(V_{C}-V_{D}\right)\right] .
$$

This correspondence follows from the reflection symmetry in the plane normal to the source-drain direction which cuts the channel in the middle. ${ }^{6}$ Therefore, the output optical power of our device, which is proportional to $I_{C}^{\mathrm{RST}}$ and is controlled by the heating bias $V_{D S}$, depends only on the magnitude of $V_{D S}$ and is invariant if the potentials on the source and drain terminals are interchanged. Thus the device exhibits an exclusive-OR dependence of the emitted light power on the input voltages $V_{S}$ and $V_{D}$, regarded as binary (high/low) logic signals.

Fig. 8 demonstrates logic operation of the complementary CHINT at three different temperatures. The collector bias is fixed at $V_{C}=3.0 \mathrm{~V}$ and the input voltages are varied from low $=0$ to high $=1.5 \mathrm{~V}$. Operation of an optical xor gate is illustrated in Fig. 8(a). The data shown were obtained in dc measurements; no attempt was made to characterize the frequency response, as our device had not been designed for a fast operation. We see that the light-output power $P_{m}$ obeys $P_{m}=$ xor $\left(V_{S}, V_{D}\right)$ at all temperatures, including room temperature. The slight asymmetry between the on states (1-0) and (0-1) indicates that our device is not perfectly symmetric due to processing variations. On the other hand, the difference between the

\footnotetext{
${ }^{5}$ We believe such an observation has never been made before. In addition to our present device, we have re-analyzed the available data measured in previously reported unipolar charge injection transistors. To our surprise, we found that most (but not all) steps in $I_{D}, I_{C}$, and $I_{S}=I_{D}+I_{C}$ are accompanied by a continuous variation in the total input power. Typically, only the slope $W_{b x}\left(V_{D}\right)$ changes at the transition. We believe this phenomenon deserves further study, both experimental and theoretical. It may shed light on the nature of switching transitions between different branches of intrinsically complicated trajectories [16] in the device phase space.

${ }^{6}$ Of course, a similar relation exists between internal states $s\left[V_{D}, V_{G}\right]$ in a field-effect transistor. The difference is that the CHINT collector $C$ is the output terminal and the symmetry expressed by (7) implies that the polarity of output current is independent of the polarity of the input voltage $V_{D}$.
} 


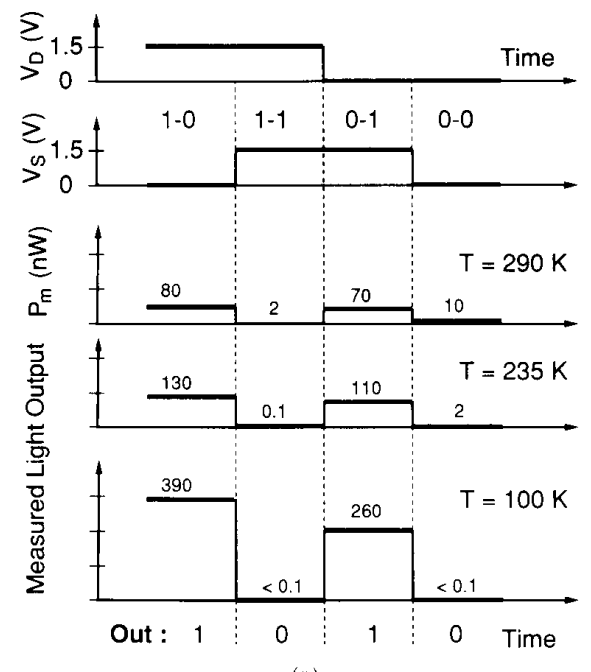

(a)

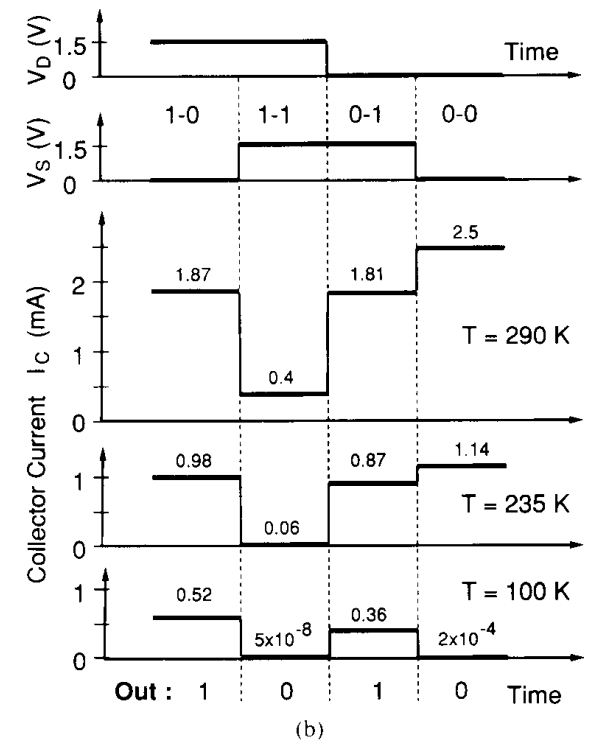

Fig. 8. Comparison of the optical and electrical logic operation of the complementary CHINT at different temperatures $T$. Four logic states $(1-0),(1-1),(0-1)$, and $(0-0)$ correspond to the electrical inputs $\left(V_{S}-V_{b}\right)$ taking the values $V=0(\operatorname{logic}-0)$ and $V=1.5 \mathrm{~V}$ (logic-1). (a) Optical output $P_{m}$ corresponds to an exclusive-OR, xor $\left(V_{S}, V_{D}\right)$. at all $T$. including room temperature. (b) Electrical output $I_{(}$is xor $\left(V_{S}, V_{D}\right)$ only at cryogenic temperatures.

off states (0-0) and (1-1) at high temperatures is owing to a nonvanishing radiation associated with the leakage current.

It should be stressed that the low radiative efficiency associated with the leakage current is crucial for a successful xor operation of our device. For comparison, Fig. 8(b) shows the electrical behavior of our device at similar temperatures and biasing conditions. While the approximate symmetry between the on states $(1-0)$ and $(0-1)$ is well maintained, the function $I_{C}=$ xor $\left(V_{S}, V_{D}\right)$ obtains only at cryogenic temperatures. At higher $T$, the leakage of holes in the state (0-0) makes it effectively an on state and the resulting function looks more like a nand than a xor.

\section{Conclusion}

The charge injection transistor has been implemented for the first time with the collector and the emitter layers of complementary conductivity type. Application of a lateral field to the n-type emitter channel gives rise to a luminescence signal resulting from the recombination of real-space transferred electrons with holes in the p-type collector layers. We employed the InGaAs/InAlAs heterostructure lattice-matched to InP. In this material, the conduction-band discontinuity is larger than that in the valence band and, therefore, in the absence of RST most of the current is due to an injection of holes from the collector. The light-emitting device operates at room temperature and exhibits a high radiative efficiency of RST electrons as well as a high light-power on/off ratio. The radiative efficiency is ensured by the design of an active collector layer where the injected minority carriers are spatially confined.

In accordance with theoretical predictions [5], the transistor generates an optical signal that is an exclusive-OR function of the voltage inputs applied to the emitter electrodes. $^{7}$ We found that the optical logic performance of our device is superior to its electrical performance, especially at higher temperatures. This difference is thoroughly investigated and shown to arise from a strong asymmetry in the radiative efficiency between electrons real space transferred into the collector and holes injected into the emitter channel. In contrast to the RST electrons, holes recombine nonradiatively-most probably in the emitter contacts. This property, as well as the high internal quantum efficiency, comparable to that in conventional long-wavelength light-emitting diodes, may lead to a variety of applications. In particular, the development of a real-space transfer laser operating as a high-speed xor logic gate, appears to be within reach.

\section{ACKNOWLEDGMENT}

The authors wish to thank E. A. Laskowski for help in selective chemical etching and D. A. Humphrey for $\mathrm{Si}_{3} \mathrm{~N}_{4}$ deposition.

\section{REFERENCES}

11] Z. S. Gribnikov. "Negative differential conductivity in a multilayer heterostructure." Fir. Tekh. Poluprovodn., vol. 6, p. 1380.1972. (Sor. Phys.-Semicond, vol. 6, p. 1204, 1973.)

12] K. Hess, H. Morkoc, H. Shichijo, and B. G. Streetman, "Negative differential resistance through real-space electron transfer." Appl. Phys. Lett., vol. 35, p. $469,1979$.

[3] S. Luryi, A. Kastalsky, A. C. Gossard, and R. H. Hendel, "Charge injection transistor based on real space hot-electron transfer, "IEEE Trans. Electron Devices, vol. ED-31, pp. 832-839, 1984

${ }^{7}$ (Note added in proof:) In a further development of this work, a multiterminal complementary RST device "ORNAND' has been implemented (Mastrapasqua et al., in IEDM Tech. Dig., 1992) showing such logic functions as or and nand in both the output light and current, with the possibility of electrically switching the nature of the gate between these two functions. 
[4] S. Luryi, "Charge injection transistors and logic circuits," Superlatt. Microstr., vol. 8, pp. 395-404, 1990.

[5] — , "Light emitting devices based on the real-space-transfer of hot electrons," Appl. Phys. Lett., vol. 58, pp. 1727-1729, 1991

[6] M. Mastrapasqua, F. Capasso, S. Luryi, A. L. Hutchinson, D. L. Sivco, and A. Y. Cho, "Light emitting charge injection transistor with p-type collector," Appl. Phys. Lett., vol. 60, pp. 2415-2417, 1992

[7] P. M. Mensz, S. Luryi, A. Y. Cho, D. L. Sivco, and F. Ren, "Real space transfer in three-terminal InGaAs/InAlAs/InGaAs heterostructure devices," Appl. Phys. Lett., vol. 56, pp. 2563-2565, 1990.

[8] P. M. Mensz, P. A. Garbinski, A. Y. Cho, D. L. Sivco, and S. Luryi, "High transconductance and large peak-to-valley ratio of negative differential conductance in three-terminal InGaAs/InAlAs realspace transfer devices," Appl. Phys. Lett., vol. 57, pp. 2558-2560, 1990.

[9] R. People, K. Wecht, K. Alavi, and A. Y. Cho, "'Measurement of the conduction-band discontinuity of molecular beam epitaxial grown $\mathrm{In}_{0,52} \mathrm{Al}_{0,48} \mathrm{As} / \mathrm{In}_{0.53} \mathrm{Ga}_{0.47} \mathrm{As} \mathrm{N}-\mathrm{n}$ heterojunction by $\mathrm{C}-\mathrm{V}$ profiling," Appl. Phys. Lett., vol. 43, pp. 118-120, 1983.

[10] S. Luryi and A. Kastalsky, "Hot electron injection devices," Superlatt. Microstr., vol, 1, pp. 389-400, 1985

[11] G. P. Agrawal and N. K. Dutta, Long-Wavelength Semiconductor Lasers. New York: Van Nostrand Reinhold, 1986.

[12] S. Luryi, "Hot-electron transistors," in High-Speed Semiconductor Devices, S. M. Sze, Ed. New York: Wiley-Interscience, 1990, ch.

[13] K. Kash and J. Shah, "Carrier energy relaxation in $\mathrm{In}_{0.53} \mathrm{Ga}_{0.47} \mathrm{As}$ determined from picosecond luminescence studies," Appl. Phys. Lett., vol. 45, pp. 401-403, 1984.

[14] I. C. Kizilyalli, K. Hess, T. Higman, M. Emanuel, and J. J. Coleman, "Ensemble Monte Carlo simulation of real space transfer (NERFET/CHINT) devices,"' Solid-State Electron., vol. 31, p. 355, 1988.

I. C. Kizillyalli and K. Hess, "Physics of real-space transfer transistors," J. Appl. Phys., vol. 65, pp. 2005-2013, 1989

[15] S. Luryi and M. R. Pinto, "Broken symmetry and the formation of hot-electron domains in real-space transfer transistors," Phys. Rev. Lett., vol. 67, pp. 2351-2354, 1991.

-. "Symmetry of the real-space transfer and collector-controlled states in charge injection transistors," Semicond. Sci Technol., vol. 7, pp. B520-B526, 1992.

[16] M. R. Pinto and S. Luryi, "Simulation of multiply connected currentvoltage characteristics in charge injection transistors," in IEDM Tech. Dig., 1991, pp. 507-510.

[17] S. Luryi, P. Mensz, M. Pinto, P. A. Garbinski, A. Y. Cho, and D. L. Sivco, "Charge injection logic," Appl. Phys. Lett., vol. 57, pp. 1787-1789, 1990.

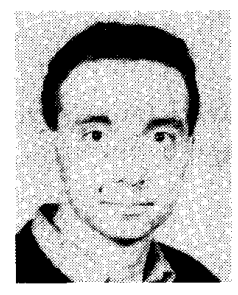

Marco Mastrapasqua (M'91) was born in Italy on February 23, 1964. He received the Doctor of Nuclear Engineering degree (summa cum laude) from the Politecnico di Milano, Milano, Italy.

During 1990, he worked at the Center for Quantum Electronics and Electronics Instrumentation of the CNR in Milano. His studies focused on dynamics of avalanche breakdown in silicon $p-n$ junction. In 1990 he was awarded the "Ercole De Castro" Fellowship by the Italian Electrotechni$\mathrm{cal}$ and Electronic Association (AEI). Since 1991. he has been a post-doctoral Member of the Technical Staff at AT\&T Bell Laboratories, Murray Hill, NJ.

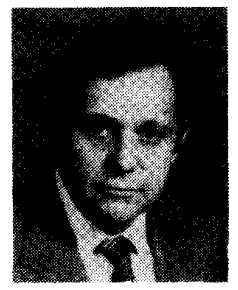

Serge Luryi (M'81-SM'85-F'90) received the Ph.D. degree in physics from the University of Toronto, Toronto, Ont. Canada, in 1978

Since 1980 , he has been with AT\&T Bell Laboratories, Murray Hill, NJ, where he is currently a Distinguished Member of the Technical Staff in the Optoelectronic Device Research Department. His main research interests are in the physics of exploratory semiconductor devices.

During 1986-1990, Dr. Luryi was the Editor of IEEE TRANSACTIONS ON ELECTRON DEVICES

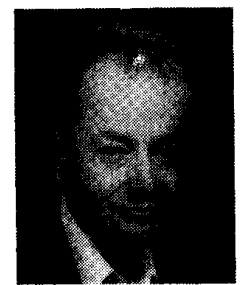

Federico Capasso (M'79-SM'85-F'87) received the doctorate degree magna cum laude in physics from the University of Rome, Rome, Italy, in 1973.

He joined AT\&T Bell Laboratories as a Member of the Technical Staff in 1977 . Since 1987 he has been head of the Quantum Phenomena and Device Research Department. He is a recipient of the 1991 IEEE David Sarnoff Award, the 1984 AT\&T Distinguished Member of Technical Staff Award, and the 1984 Award of Excellence of the Society for Technical Communication. He has co-authored over $150 \mathrm{pa}$ pers, given over ninety invited talks at international conferences, and holds 20 U.S. patents.

Dr Capasso is a member of the American Physical Society, the Optical Society of America, the American Association for the Advancement of Science, and the International Society for Optical Engineering. He is listed in Who's Who in America, Who's Who in Engineering, and American Men and Women in Science.

Albert L. Hutchinson, photograph and biography not available at the time of publication.

Deborah L. Sivco, photograph and biography not available at the time of publication.

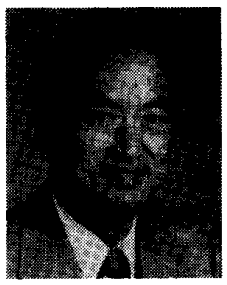

Alfred Y. Cho (S'57-M'60-SM'79-F'81) received the B.S., M.S., and Ph.D. degrees in electrical engineering from the University of Illinois. Urbana, in 1960, 1961, and 1968, respectively.

$\mathrm{He}$ is Director of the Materials Processing Research Laboratory at AT\&T Bell Laboratories, Murray Hill, NJ. He joined Bell Laboratories in 1968 where he developed a crystal growth technology called molecular-beam epitaxy (MBE). His many significant research accomplishments include the construction of surface phase diagram for MBE crystal growth the first fabrication of an MBE artificial superlattice, the first MBE IMPATT diode, mixer diode, and field-effect transistor operating at microwave frequencies, and the first MBE double-heterostructure laser operating $\mathrm{CW}$ at room temperature. His recent work is in the area of quantum-well devices. He has published over 250 papers in surface physics, crystal growth, and device physics and performance. He holds 43 patents on crystal growth and semiconductor devices related to MBE.

Dr. Cho is a recipient of the Electronics Division Award of the Electrochemical Society (1977), the American Physical Society International Prize for New Materials (1982), the IEEE Morris N. Liebmann Award (1982). the University of Illinois Electrical and Computer Engineering Distinguished Alumnus Award (1985), the Chinese Institute of Engineers USA Distinguished Achievement Award (1985), the GaAs Symposium AwardFord (1986), the Heinrich Welker Medal-Siemens (1986), the Solid State Science and Technology Medal of the Electrochemical Society (1987), the College of Engineering Alumni Honor Award of the University of Illinois (1988), the World Materials Congress Award of ASM International (1988), the Gaede-Langmuir Award of the American Vacuum Society (1988), and the Industrial Research Institute Achievement Award of the Industrial Research Institute, Inc. (1988). He is a Fellow of the American Physical Society, and the American Academy or Arts and Sciences. He is a member of the American Vacuum Society, the Electrochemical Society, the Materials Research Society, the National Academy of Engineering, and the $\mathrm{Na}$ tional Academy of Sciences. 